\title{
Some variants of Wardowski fixed point theorem
}

\author{
Muhammad Nazam ${ }^{1}$, Hassen Aydi ${ }^{2,3}$, , Choonkil Park ${ }^{5 *}$, Muhammad Arshad ${ }^{6}$, Ekrem Savas ${ }^{7}$ and \\ Dong Yun Shin ${ }^{8 *}$
}

"Correspondence:

baak@hanyang.ac.kr;

dyshin@uos.ac.kr

${ }^{5}$ Research Institute for Natural

Sciences, Hanyang University, Seoul 04763, Korea

${ }^{8}$ Department of Mathematics,

University of Seoul, Seoul 02504,

Korea

Full list of author information is

available at the end of the article

\begin{abstract}
The purpose of this paper is to consider some F-contraction mappings in a dualistic partial metric space and to provide sufficient related conditions for the existence of a fixed point. The obtained results are extensions of several ones existing in the literature. Moreover, we present examples and an application to support our results.
\end{abstract}

MSC: $47 \mathrm{H} 09 ; 47 \mathrm{H} 10 ; 54 \mathrm{H} 25$

Keywords: Fixed point; Dualistic partial metric; F-contraction

\section{Introduction}

The study of metric fixed point theory was initiated by Banach (1922). It has been enriched by introducing several structures, generalizing underlying metric space, and the contractive condition. The Banach contraction principle has been proved in several different abstract metric spaces (see [1-10]). Before the publication of the paper of Mustafa et al. [5], the metric functions were defined on $X^{2}$, Mustafa et al. [5] introduced a metric function defined on $X^{3}$, and it is known as a $G$-metric. Mustafa et al. [5] proved the Banach contraction principle (BCP) in G-metric spaces and gave several examples to establish its superiority over the BCP in metric spaces. Sedghi et al. [11] introduced another abstract metric defined on $X^{3}$, called an $S$-metric, and proved the analogue of the BCP.

Working on network topologies in computer science, Matthews [12] observed that the self-distance of a point need not be zero. This led him to introduce a partial metric and hence obtain an analogue of the BCP. Later, many researchers worked on partial metric spaces and produced several papers on partial metric topologies and existence of fixed points of self-mappings (see [13-17]).

Neill [18] observed during his study on dual topologies that a partial metric may have negative value, that is, the range set of a partial metric can be considered as a real line. It is known as a dualistic partial metric. Oltra et al. [19] presented an analogue of the Banach fixed point result in complete dualistic partial metric spaces using some convergence properties of sequences. Subsequently, Nazam et al. [20-22] and Pitea [23] published some papers addressing the existence of fixed points of self-mappings defined on dualistic partial metric spaces.

(c) The Author(s) 2021. This article is licensed under a Creative Commons Attribution 4.0 International License, which permits use sharing, adaptation, distribution and reproduction in any medium or format, as long as you give appropriate credit to the original author(s) and the source, provide a link to the Creative Commons licence, and indicate if changes were made. The images or other third party material in this article are included in the article's Creative Commons licence, unless indicated otherwise in a credit line to the material. If material is not included in the article's Creative Commons licence and your intended use is not permitted by statutory regulation or exceeds the permitted use, you will need to obtain permission directly from the copyright holder. To view a copy of this licence, visit http://creativecommons.org/licenses/by/4.0/. 
The concept of $F$-contractions given by Wardowski [24] has attracted people in fixed point theory, and numerous research papers on $F$-contractions have been published (see [25-37] and the references therein). Proinov(2020) [38] showed that some fixed point theorems of Wardowski (2012) [24], Jleli and Samet (2014) [3] that have recently appeared are equivalent to a special case of the well-known fixed point theorem of Skof (1977) [39].

In this paper, we investigate fixed points of $F$-contractions under the rules practiced in dualistic partial metric spaces. We also discuss how this differs from the classical $F$ contraction introduced by Wardowski [24].

\section{Preliminaries}

Let $T: \mho \rightarrow \mho$ be a self-mapping on a nonempty set $\mho$. The sequence $\left\{\ell_{n}\right\}$ in $\mho$ with an initial point $\ell_{0}$ given as

$$
\ell_{n}=T\left(\ell_{n-1}\right) \quad \text { for all } n \in \mathbb{N}
$$

is said to be a Picard iterative sequence. The partial metric space was defined by Matthews [12].

Definition 1 ([12]) A partial metric on a nonempty set $\mho$ is a function $P: \mho \times \mho \rightarrow[0, \infty)$ such that

$\left(P_{1}\right) \ell=\hbar \Leftrightarrow P(\ell, \ell)=P(\hbar, \hbar)=P(\ell, \hbar)$

(P) $P(\ell, \ell) \leq P(\ell, \hbar)$;

$\left(P_{3}\right) P(\ell, \hbar)=P(\hbar, \ell)$

$\left(P_{4}\right) P(\ell, v) \leq P(\ell, \hbar)+P(\hbar, v)-P(\hbar, \hbar)$

for all $\ell, \hbar, v \in \mho$.

The notion of a partial metric was extended by Neill [18] in the following.

Definition 2 ([18]) A dualistic partial metric on a nonempty set $\mho$ is a function $\mathcal{V}: \mho \times$ $\mho \rightarrow \mathbb{R}$ fulfilling the following properties for all $\ell, \hbar, v \in \mho$ :

$\left(\mathcal{V}_{1}\right) \quad \ell=\hbar \Leftrightarrow \mathcal{V}(\ell, \ell)=\mathcal{V}(\hbar, \hbar)=\mathcal{V}(\ell, \hbar)$

$\left(\mathcal{V}_{2}\right) \mathcal{V}(\ell, \ell) \leq \mathcal{V}(\ell, \hbar)$

$\left(\mathcal{V}_{3}\right) \mathcal{V}(\ell, \hbar)=\mathcal{V}(\hbar, \ell)$

$\left(\mathcal{V}_{4}\right) \mathcal{V}(\ell, v) \leq \mathcal{V}(\ell, \hbar)+\mathcal{V}(\hbar, v)-\mathcal{V}(\hbar, \hbar)$

Then $(\mho, \mathcal{V})$ is said to be a dualistic partial metric space.

Remark 1 Each partial metric is a dualistic partial metric. The converse does not hold in general. For this, consider

$$
\mathcal{V}_{m}: \mathbb{R} \times \mathbb{R} \rightarrow \mathbb{R}, \quad \mathcal{V}_{m}(\ell, \hbar)=\max \{\ell, \hbar\} \quad \forall \ell, \hbar \in \mathbb{R}
$$

Then $\mathcal{V}_{m}$ is a dualistic partial metric (see [21]). Here $\mathcal{V}_{m}$ is not a partial metric since for any $\ell<0, \hbar<0$ it implies $\mathcal{V}_{m}(\ell, \hbar)<0$.

Note that in a dualistic partial metric, $\mathcal{V}(\ell, \hbar)=0$ does not necessarily imply $\ell=\hbar$. Indeed, $\mathcal{V}_{m}(-1,0)=0$. This case creates a problem in obtaining a fixed point of a selfmapping in a dualistic partial metric space. To resolve this problem, in this paper we em- 
ploy our proposed $F$-contraction (defined in the next section) along with axioms $\left(\mathcal{V}_{2}\right)$ and $\left(\mathcal{V}_{1}\right)$ to get a fixed point.

We give now some examples of dualistic partial metrics. The first is related to the classic one.

Example 1 If $(\mho, d)$ is a metric space and $c \in \mathbb{R}$ is an arbitrary constant, then

$$
\mathcal{V}(\ell, \hbar)=d(\ell, \hbar)+c
$$

is a dualistic partial metric on $\mho$.

Next, we use a partial metric to define a dualistic partial metric space.

Example $2([18])$ Let $(\mho, P)$ be a partial metric space. The mapping $\mathcal{V}: \mho \times \mho \rightarrow \mathbb{R}$ given as

$$
\mathcal{V}(\ell, \hbar)=P(\ell, \hbar)-P(\ell, \ell)-P(\hbar, \hbar) \quad \text { for all } \ell, \hbar \in \mho
$$

verifies axioms $\left(\mathcal{V}_{1}\right)-\left(\mathcal{V}_{4}\right)$. So it is dualistic partial metric on $\mho$. We emphasize that $\mathcal{V}(\ell, \hbar)$ may have negative values.

Example 3 ([22]) Let $\mho=\mathbb{R}$. Consider $\mathcal{V}: \mho \times \mho \rightarrow \mathbb{R}$ as

$$
\mathcal{V}(\ell, \hbar)= \begin{cases}|\ell-\hbar| & \text { if } \ell \neq \hbar, \\ -b & \text { if } \ell=\hbar ; b>0\end{cases}
$$

Then $(\mho, \mathcal{V})$ is a dualistic partial metric space.

Neill [18] ensured that each dualistic partial metric $\mathcal{V}$ on $\mho$ generates a $T_{0}$ topology $\tau[\mathcal{V}$ ] on $\mho$ having as a base the family of $\mathcal{V}$-balls $\left\{B_{\mathcal{V}}(\ell, \omega): \ell \in \mho, \omega>0\right\}$, where

$$
B_{\mathcal{V}}(\ell, \omega)=\{\hbar \in \mho: \mathcal{V}(\ell, \hbar)<\omega+\mathcal{V}(\ell, \ell)\}
$$

If $(\mho, \mathcal{V})$ is a dualistic partial metric space, then the function

$$
d^{*}: \mho \times \mho \rightarrow \mathbb{R}_{0}^{+}, \quad d^{*}(\ell, \hbar)=\mathcal{V}(\ell, \hbar)-\mathcal{V}(\ell, \ell)
$$

is a quasi-metric on $\mho$ such that $\tau(\mathcal{V})=\tau\left(d_{\mathcal{V}}^{s}\right)$ and $d_{\mathcal{V}}^{s}(\ell, \hbar)=\max \left\{d^{*}(\ell, \hbar), d^{*}(\hbar, \ell)\right\}$ is a metric on $\mho$. It is said to be an induced metric on $(\mho, \mathcal{V})$.

The following describes the convergence criteria in a dualistic partial metric space established by Oltra et al. [19].

Definition 3 ([19]) Let $\left\{\ell_{n}\right\}_{n \in \mathbb{N}}$ be a sequence in the dualistic partial metric space $(\mho, \mathcal{V})$.

(1) $\left\{\ell_{n}\right\}$ is convergent to an element $\ell$ in $(\mho, \mathcal{V})$ if

$$
\lim _{n \rightarrow \infty} \mathcal{V}\left(\ell_{n}, \ell\right)=\mathcal{V}(\ell, \ell)
$$


(2) $\left\{\ell_{n}\right\}$ in $(\mho \delta, \mathcal{V})$ is said to be Cauchy if

$$
\lim _{n, m \rightarrow \infty} \mathcal{V}\left(\ell_{n}, \ell_{m}\right) \quad \text { exists and is finite. }
$$

(3) $(\mathcal{V}, \mathcal{V})$ is said to be complete if every Cauchy sequence $\left\{\ell_{n}\right\}_{n \in \mathbb{N}}$ in $\mho$ converges, with respect to $\mathcal{T}[\mathcal{V}]$, to an element $\ell \in \mho$ such that

$$
\mathcal{V}(\ell, \ell)=\lim _{n, m \rightarrow \infty} \mathcal{V}\left(\ell_{n}, \ell_{m}\right)
$$

Remark 2 For a sequence $\left\{\ell_{n}\right\} \subset \mho$, the convergence with respect to an induced metric space $\left(\mho, d_{\mathcal{V}}^{s}\right)$ may not lead to the convergence with respect to the dualistic partial metric space $(\mho, \mathcal{V})$.

Indeed, consider $\left\{\ell_{n}=\frac{1}{n}-1\right\} \subset \mho$ and take

$$
\mathcal{V}: \mho \times \mho \rightarrow \mathbb{R}, \quad \mathcal{V}(\ell, \hbar)= \begin{cases}|\ell-\hbar|=d_{\mathcal{V}}^{s}(\ell, \hbar) & \text { if } \ell \neq \hbar \\ -1 & \text { if } \ell=\hbar\end{cases}
$$

Clearly,

$$
\lim _{n \rightarrow \infty} d_{\mathcal{V}}^{s}\left(\ell_{n},-1\right)=0
$$

This implies that $\ell_{n} \rightarrow-1$ with respect to $\left(\mho, d_{\mathcal{V}}^{s}\right)$. On the other hand, consider

$$
\lim _{n \rightarrow \infty} \mathcal{V}\left(\ell_{n},-1\right)=\mathcal{V}(-1,-1)
$$

That is,

$$
\lim _{n \rightarrow \infty}\left|\ell_{n}+1\right|=-1
$$

It is a contradiction. Thus, $\ell_{n} \nrightarrow-1$ with respect to $(\mho, \mathcal{V})$.

With regard to the connections between convergence and Cauchyness in dualistic partial metric spaces, we recall the next lemma.

Lemma $1([19$, Lemma 2.2]) Let $(\mho, \mathcal{V})$ be a dualistic partial metric space.

(1) Each Cauchy sequence in $\left(\mho, d_{\mathcal{V}}^{s}\right)$ is also Cauchy in $(\mho, \mathcal{V})$.

(2) $(\mho, \mathcal{V})$ is complete iff the induced metric space $\left(\mho, d_{\mathcal{V}}^{s}\right)$ is complete.

(3) A sequence $\left\{\ell_{n}\right\}$ in $\mho$ converges to an element $v \in \mho$ with respect to $\mathcal{T}\left[\left(d_{\mathcal{V}}^{s}\right)\right]$ if and only if

$$
\lim _{n \rightarrow \infty} \mathcal{V}\left(v, \ell_{n}\right)=\mathcal{V}(v, v)=\lim _{n, m \rightarrow \infty} \mathcal{V}\left(\ell_{n}, \ell_{m}\right)
$$

\section{Main results}

Definition 4 ([24]) Denote by $\mathcal{F}$ the set of all functions $F:(0, \infty) \rightarrow \mathbb{R}$ fulfilling the properties:

(1) $F$ is strictly increasing; 
(2) For any sequence of positive terms $\left\{a_{n}\right\}, \lim _{n \rightarrow \infty} a_{n}=0 \Longleftrightarrow \lim _{n \rightarrow \infty} F\left(a_{n}\right)=-\infty$;

(3) There is $k$ in $(0,1)$ such that $\lim _{\alpha \rightarrow 0^{+}} \alpha^{k} F(\alpha)=0$.

Example 4 The following functions $F:(0, \infty) \rightarrow \mathbb{R}$ given for all $\ell \in(0, \infty)$ as:

(i) $F(\ell)=\ln (\ell)$,

(ii) $F(\ell)=\ell+\ln (\ell)$,

(iii) $F(\ell)=\ln \left(\ell^{2}+\ell\right)$,

(iv) $F(\ell)=-\frac{1}{\sqrt{\ell}}$,

belong to the set $\mathcal{F}$.

In [24], Wardowski introduced a remarkable contraction known as $F$-contraction and presented a method to obtain fixed points of such contractions in complete metric spaces. The following theorem is the variant of Wardowski's fixed point theorem [24] in the complete dualistic partial metric spaces. Theorem 1 is a useful generalization of the mentioned theorem.

Theorem 1 Let $(M, \mathcal{V})$ be a complete dualistic partial metric space, $F \in \mathcal{F}$, and $T: M \rightarrow$ $M$ be a continuous mapping for which there exists $\tau>0$ such that, for all $j, k$ from $M$, the following implication holds:

$$
\mathcal{V}(T j, T k) \neq 0 \quad \Longrightarrow \quad \tau+F(|\mathcal{V}(T j, T k)|) \leq F(|\mathcal{V}(j, k)|)
$$

Then $T$ possesses a unique fixed point.

Proof Let $j_{0}$ be an element from $M$ and $j_{n+1}=T j_{n}$ for all $n \in \mathbb{N}$. If there is $n_{o} \in \mathbb{N}$ for which $j_{n_{0}+1}=j_{n_{o}}$, then $j_{n_{o}}$ is a fixed point of $T$. Therefore, we may assume that $j_{n} \neq j_{n+1}$ for any $n \in \mathbb{N}$. Given that $\mathcal{V}\left(j_{n}, j_{n+1}\right) \neq 0$ for any $n \in \mathbb{N}$. By the use of the contractive condition, we obtain, for $n \geq 1$,

$$
F\left(\left|\mathcal{V}\left(j_{n}, j_{n+1}\right)\right|\right) \leq F\left(\left|\mathcal{V}\left(j_{n-1}, j_{n}\right)\right|\right)-\tau<F\left(\left|\mathcal{V}\left(j_{n-1}, j_{n}\right)\right|\right)
$$

which, by taking advantage of the monotonicity of $F,\left\{\left|\mathcal{V}\left(j_{n}, j_{n+1}\right)\right|\right\}$ is decreasing. Let $r \geq 0$ be its limit. By contractive condition (3.1), we have

$$
F\left(\left|\mathcal{V}\left(j_{n+1}, j_{n}\right)\right|\right)=F\left(\left|\mathcal{V}\left(T\left(j_{n}\right), T\left(j_{n-1}\right)\right)\right|\right) \leq F\left(\left|\mathcal{V}\left(j_{n}, j_{n-1}\right)\right|\right)-\tau
$$

Thus

$$
F\left(\left|\mathcal{V}\left(j_{n+1}, j_{n}\right)\right|\right) \leq F\left(\left|\mathcal{V}\left(j_{n}, j_{n-1}\right)\right|\right)-\tau
$$

This in turns implies that

$$
F\left(\left|\mathcal{V}\left(j_{n+1}, j_{n}\right)\right|\right) \leq F\left(\left|\mathcal{V}\left(j_{1}, j_{0}\right)\right|\right)-n \tau
$$

Letting $n \rightarrow \infty$ and using $\left(F_{2}\right)$, we get

$$
\lim _{n \rightarrow \infty} F\left(\left|\mathcal{V}\left(j_{n+1}, j_{n}\right)\right|\right)=-\infty \Longleftrightarrow \lim _{n \rightarrow \infty}\left|\mathcal{V}\left(j_{n+1}, j_{n}\right)\right|=0
$$


This yields that

$$
\lim _{n \rightarrow \infty} \mathcal{V}\left(j_{n+1}, j_{n}\right)=0=r
$$

Focusing now on the self-distance, we have, for $n \in \mathbb{N}$,

$$
F\left(\left|\mathcal{V}\left(j_{n+1}, j_{n+1}\right)\right|\right) \leq F\left(\left|\mathcal{V}\left(j_{n}, j_{n}\right)\right|\right)-\tau<F\left(\left|\mathcal{V}\left(j_{n}, j_{n}\right)\right|\right)
$$

and so $\left\{\left|\mathcal{V}\left(j_{n}, j_{n}\right)\right|\right\}$ is a sequence decreasing to some $l \geq 0$. Arguing as above, we get that $l=0$. By the third property of functions in $\mathcal{F}$, there is $\theta \in(0,1)$ such that $\lim _{n \rightarrow \infty}\left|\mathcal{V}_{n}\right|^{\theta} \times$ $F\left(\left|\mathcal{V}_{n}\right|\right)=0$.

Consider $\mathcal{V}_{n}=\mathcal{V}\left(j_{n}, j_{n+1}\right)$ for $n \in \mathbb{N}$. By applying repeatedly inequality (3.2), it follows that

$$
F\left(\left|\mathcal{V}_{n}\right|\right) \leq F\left(\left|\mathcal{V}_{0}\right|\right)-n \tau
$$

or

$$
\left|\mathcal{V}_{n}\right|^{\theta} F\left(\left|\mathcal{V}_{n}\right|\right) \leq\left|\mathcal{V}_{n}\right|^{\theta}\left(F\left(\left|\mathcal{V}_{0}\right|\right)-n \tau\right), \quad n \in \mathbb{N}
$$

Letting $n \rightarrow \infty$ and taking advantage of the properties of the function $F$, we get that $n\left|\mathcal{V}_{n}\right|^{\theta} \rightarrow 0$ as $n \rightarrow \infty$. There is $N_{1} \in \mathbb{N}$ such that

$$
\left|\mathcal{V}_{n}\right| \leq \frac{1}{n^{\frac{1}{\theta}}}, \quad n \geq N_{1}
$$

Denote by $\mathcal{V}_{n}=\mathcal{V}\left(j_{n}, j_{n}\right)$ for $n \in \mathbb{N}$. Similarly, there is $N_{2} \in \mathbb{N}$ such that, for any $n \geq N_{2}$,

$$
\left|\mathcal{V}_{n}\right| \leq \frac{1}{n^{\frac{1}{\theta}}}
$$

Having in mind inequalities (3.5) and (3.6), we have, for $m>n \geq \max \left\{N_{1}, N_{2}\right\}$,

$$
\begin{aligned}
d^{*}\left(j_{n}, j_{m}\right) & \leq \sum_{\ell=0}^{m-n-1} d^{*}\left(j_{n+\ell}, j_{n+\ell+1}\right) \\
& \leq \sum_{\ell=0}^{m-n-1}\left(\left|\mathcal{V}\left(j_{n+\ell}, j_{n+\ell+1}\right)\right|+\left|\mathcal{V}\left(j_{n+\ell}, j_{n+\ell}\right)\right|\right) \\
& \leq 2 \sum_{\ell=0}^{m-n-1} \frac{1}{\ell^{\frac{1}{\theta}}}
\end{aligned}
$$

Taking the limit to $\infty$, it follows that $\left\{d^{*}\left(j_{n}, j_{m}\right)\right\} \rightarrow 0$. Applying an analogous procedure, we get that $\left\{d^{*}\left(j_{m}, j_{n}\right)\right\} \rightarrow 0$, hence $d_{\mathcal{V}}^{s}\left(j_{n}, j_{m}\right) \rightarrow 0$, so $\left\{j_{n}\right\}$ is a Cauchy sequence in the complete metric space $\left(M, d_{\mathcal{V}}^{s}\right)$. Let $j$ be its limit. Obviously,

$$
\lim _{n \rightarrow \infty} \mathcal{V}\left(j_{n}, j\right)=\mathcal{V}(j, j)=0, \quad \lim _{n, m \rightarrow \infty} \mathcal{V}\left(j_{n}, j_{m}\right)=0
$$

Since $T$ is a continuous mapping, $\left\{T j_{n}\right\}$ converges to $T j$. This implies that $\mathcal{V}\left(T j_{n}, T j_{n}\right) \rightarrow$ $\mathcal{V}(T j, T j)$. Since $\mathcal{V}\left(T j_{n}, T j_{n}\right) \rightarrow \mathcal{V}(j, j)=0$, it follows that $\mathcal{V}(T j, T j)=0$. 
In view that

$$
\mathcal{V}(j, T j) \leq \mathcal{V}\left(j, j_{n+1}\right)+\mathcal{V}\left(j_{n+1}, T j\right)-\mathcal{V}\left(j_{n+1}, j_{n+1}\right), \quad n \in \mathbb{N},
$$

and

$$
\mathcal{V}\left(j_{n+1}, T j\right) \leq \mathcal{V}\left(j_{n+1}, j\right)+\mathcal{V}(j, T j)-\mathcal{V}(j, j), \quad n \in \mathbb{N},
$$

by considering $n \rightarrow \infty$ and using the continuity of $T$, we obtain $\mathcal{V}(j, T j)=0$.

Thus $\mathcal{V}(T j, T j)=\mathcal{V}(j, j)=\mathcal{V}(j, T j)$, so $T j=j$. Let us focus now on the uniqueness of the fixed point of $T$. Assume that $j$ and $k$ are two distinct fixed points of $T$. If $\mathcal{V}(j, k) \neq 0$, then the following relations hold true:

$$
F(|\mathcal{V}(j, k)|)=F(|\mathcal{V}(T j, T k)|) \leq F(|\mathcal{V}(j, k)|)-\tau
$$

which is a contradiction. Therefore, $\mathcal{V}(j, k)=0$. Similarly, it can be proved that $\mathcal{V}(j, j)=0$ and $\mathcal{V}(k, k)=0$. It follows that $j=k$, and so the fixed point is unique.

Remark 3 By setting $\mathcal{V}(j, j)=0$ for all $j \in M$, we have $\mathcal{V}(j, k) \geq 0$ for all $j, k \in M$ and thus $\mathcal{V}: M \times M \rightarrow \mathbb{R}$ is restricted to $\mathcal{V}: M \times M \rightarrow \mathbb{R}^{+}$, which is equal to the induced metric $d_{\mathcal{V}}^{s}$ on $M$. Hence Wardowski fixed point theorem [24] is a particular case of Theorem 1.

We continue with an example with respect to this theorem. Example 5 does not only explain Theorem 1, but also shows that the Wardowski fixed point theorem [24] is not applicable for $\ell=\hbar$.

Example 5 Let $\mathcal{A}=(-\infty, 0]$. Define the mapping

$$
\mathcal{V}: \mathcal{A} \times \mathcal{A} \rightarrow \mathbb{R}, \mathcal{V}(\ell, \hbar)= \begin{cases}|\ell-\hbar| & \text { if } \ell \neq \hbar \\ \ell \vee \hbar & \text { if } \ell=\hbar\end{cases}
$$

Then $(\mathcal{A}, \mathcal{V})$ is a complete dualistic partial metric space. Let $\tau>0$ and define the mapping:

$$
T: \mathcal{A} \rightarrow \mathcal{A}, T(\ell)= \begin{cases}\frac{e^{-\tau} \ell}{2} & \text { if } \ell \in \mathbb{Q} \text { (a set of rational numbers) } \\ \frac{e^{-\tau} \ell}{3} & \text { if } \ell \in \mathbb{Q}^{\prime} \text { (a set of irrational numbers). }\end{cases}
$$

Contractive condition (2.1) is satisfied for $F(\ell)=\ln (\ell)$ for all $\ell>0$. For this, consider the following:

Case 1. (a) $\ell \neq \hbar$; if $\ell, \hbar \in \mathbb{Q}$, then

$$
|\mathcal{V}(T(\ell), T(\hbar))|=\frac{1}{2} e^{-\tau}|\ell-\hbar|<e^{-\tau}|\mathcal{V}(\ell, \hbar)|,
$$

which implies $\tau+F(|\mathcal{V}(T \ell, T \hbar)|) \leq F(|\mathcal{V}(\ell, \hbar)|)$.

(b) $\ell \neq \hbar$; if $\ell, \hbar \in \mathbb{Q}^{\prime}$, then

$$
|\mathcal{V}(T(\ell), T(\hbar))|=\frac{1}{3} e^{-\tau}|\ell-\hbar|<e^{-\tau}|\mathcal{V}(\ell, \hbar)|,
$$

which leads to $\tau+F(|\mathcal{V}(T \ell, T \hbar)|) \leq F(|\mathcal{V}(\ell, \hbar)|)$. 
Case 2. (a) $\ell=\hbar$; if $\ell \in \mathbb{Q}$, then

$$
|\mathcal{V}(T(\ell), T(\hbar))|=\left|\frac{1}{2} e^{-\tau} \ell\right|<e^{-\tau}|\mathcal{V}(\ell, \hbar)|
$$

(b) $\ell=\hbar$; if $\ell \in \mathbb{Q}^{\prime}$, then

$$
|\mathcal{V}(T(\ell), T(\hbar))|=\left|\frac{1}{3} e^{-\tau} \ell\right|<e^{-\tau}|\mathcal{V}(\ell, \hbar)| .
$$

From (a) and (b), we infer that $\tau+F(|\mathcal{V}(T \ell, T \hbar)|) \leq F(|\mathcal{V}(\ell, \hbar)|)$.

Case 3. If $\ell \in \mathbb{Q}, \hbar \in \mathbb{Q}^{\prime}$ and vice versa, then

$$
|\mathcal{V}(T(\ell), T(\hbar))|=\left|\frac{\ell}{2}-\frac{\hbar}{3}\right| e^{-\tau} \leq e^{-\tau}|\mathcal{V}(\ell, \hbar)| .
$$

Thus, $\tau+F(|\mathcal{V}(T \ell, T \hbar)|) \leq F(|\mathcal{V}(\ell, \hbar)|)$. Hence, $T$ satisfies all the conditions of Theorem 1 . Note that $\ell=0$ is the unique fixed point of $T$.

The following theorem is for Kannan type F-contraction in the dualistic partial metric spaces. It is a useful generalization of [40, Theorem 2].

Theorem 2 Let $(\mho, \mathcal{V})$ be a complete dualistic partial metric space, $F \in \mathcal{F}$, and $T: \mho \rightarrow \mho$ be a continuous mapping for which there exists $\tau>0$ such that, for all $j$ and $k$ in $\mho$, the following implication holds:

$$
\mathcal{V}(T j, T k) \neq 0 \quad \Longrightarrow \quad \tau+F(|\mathcal{V}(T j, T k)|) \leq F\left(\frac{1}{2}(|\mathcal{V}(j, T j)|+|\mathcal{V}(k, T k)|)\right)
$$

Then $T$ admits a unique fixed point.

Proof For $j_{0}$ from $\mho$, set $j_{n+1}=T j_{n}$ for $n \in \mathbb{N}$. If there is $s \in \mathbb{N}$ for which $j_{s+1}=j_{s}$, then $j_{s}$ is a fixed point of $T$. We presume that $j_{n} \neq j_{n+1}$ for any $n \in \mathbb{N}$. Given $\mathcal{V}\left(j_{n}, j_{n+1}\right) \neq 0$ for any $n \in \mathbb{N}$. By the use of the contractive condition, we obtain, for $n \geq 1$,

$$
\begin{aligned}
F\left(\left|\mathcal{V}\left(j_{n}, j_{n+1}\right)\right|\right) & \leq F\left(\frac{1}{2}\left(\left|d\left(j_{n-1}, j_{n}\right)\right|+\left|\mathcal{V}\left(j_{n}, j_{n+1}\right)\right|\right)\right)-\tau \\
& <F\left(\frac{1}{2}\left(\left|d\left(j_{n-1}, j_{n}\right)\right|+\left|\mathcal{V}\left(j_{n}, j_{n+1}\right)\right|\right)\right),
\end{aligned}
$$

from which, by taking advantage of the monotony of $F$, we have that $\left\{\left|\mathcal{V}\left(j_{n}, j_{n+1}\right)\right|\right\}$ is a decreasing sequence. Let $r \geq 0$ be its limit.

By applying inequality (3.8) and keeping in mind also that $\left\{\left|\mathcal{V}\left(j_{n}, j_{n+1}\right)\right|\right\}$ is a decreasing sequence, we get, for $n \geq 1$,

$$
F\left(\left|\mathcal{V}\left(j_{n}, j_{n+1}\right)\right|\right) \leq F\left(\left|\mathcal{V}\left(j_{n-1}, j_{n}\right)\right|\right)-\tau \leq F\left(\left|\mathcal{V}\left(j_{0}, j_{1}\right)\right|\right)-n \tau
$$

It follows that $\lim _{n \rightarrow \infty} F\left(\left|\mathcal{V}\left(j_{n}, j_{n+1}\right)\right|\right)=-\infty$. Having in mind $F \in \mathcal{F}$, we obtain that $\lim _{n \rightarrow \infty}\left|\mathcal{V}\left(j_{n}, j_{n+1}\right)\right|=0$. 
Let us now analyze the self-distance. Presume that there exists $n_{0} \in \mathbb{N}$ such that $\mathcal{V}\left(j_{n}, j_{n}\right) \neq 0$ for any $n \geq n_{0}$, where $n_{0} \in \mathbb{N}$. The contractive condition imposes

$$
F\left(\left|\mathcal{V}\left(j_{n+1}, j_{n+1}\right)\right|\right) \leq F\left(\left|d\left(j_{n}, j_{n+1}\right)\right|\right)-\tau
$$

From (3.9), it follows that

$$
F\left(\left|\mathcal{V}\left(j_{n+1}, j_{n+1}\right)\right|\right) \leq F\left(\left|\mathcal{V}\left(j_{n_{0}}, j_{n_{0}+1}\right)\right|\right)-\left(n-n_{0}\right) \tau
$$

Letting $n \rightarrow \infty$ and using the properties of the function $F$, we get that $\lim _{n \rightarrow \infty} \mathcal{V}\left(j_{n}, j_{n}\right)=0$. As in the proof of Theorem 1, we obtain that $\left\{j_{n}\right\}$ is a Cauchy sequence and so it is convergent to some $j$. From the preceding lines, $\mathcal{V}(j, j)=0$. Since $T$ is continuous, $\left\{T_{j_{n}}\right\}$ converges to $T j$, and therefore $\mathcal{V}\left(T j_{n}, T j_{n}\right) \rightarrow \mathcal{V}(T j, T j)$. On the other hand, $\mathcal{V}\left(T j_{n}, T j_{n}\right) \rightarrow$ $\mathcal{V}(j, j)=0$. We have obtained that $\mathcal{V}(T j, T j)=0$.

In view that

$$
\mathcal{V}(j, T j) \leq \mathcal{V}\left(j, j_{n+1}\right)+\mathcal{V}\left(j_{n+1}, T j\right)-\mathcal{V}\left(j_{n+1}, j_{n+1}\right), \quad n \in \mathbb{N},
$$

and also that

$$
\mathcal{V}\left(j_{n+1}, T j\right) \leq \mathcal{V}\left(j_{n+1}, j\right)+\mathcal{V}(j, T j)-\mathcal{V}(j, j), \quad n \in \mathbb{N},
$$

by considering $n \rightarrow \infty$ and using the continuity of $T$, we obtain $\mathcal{V}(j, T j)=0$. We conclude that $T j=j$, and so $T$ has a fixed point.

Assume now that the set $\left\{n \in \mathbb{N}, \mathcal{V}\left(j_{n}, j_{n}\right)=0\right\}$ is not bounded. In this case, there exists a subsequence of $\left\{j_{n}\right\},\left\{j_{n_{k}}\right\}$ for which $\mathcal{V}\left(j_{n_{k}}, j_{n_{k}}\right)=0, k \in \mathbb{N}$. We repeat the same lines as in the proof of Theorem 1 for this subsequence $\left\{j_{n_{k}}\right\}$, and we obtain that $\left\{j_{n_{k}}\right\}$ is convergent to $j \in \mho$ with $\mathcal{V}(j, j)=0$. The equality $j=T j$ follows as in the case when $\left\{n \in \mathbb{N}, \mathcal{V}\left(j_{n}, j_{n}\right)=0\right\}$ is bounded.

Summing up, we have proved that $T$ has a fixed point.

Let us focus now on the uniqueness of the fixed point of $T$. Assume that $j$ and $k$ are two distinct fixed points of $T$. Now suppose that $\mathcal{V}(j, j) \neq 0$. Then the following holds:

$$
F(|\mathcal{V}(j, j)|)=F(\mathcal{V}(T j, T j)) \leq F(|\mathcal{V}(j, T j)|)-\tau=F(|\mathcal{V}(j, j)|)-\tau
$$

which is impossible. Hence, $\mathcal{V}(j, j)=0$. Similarly, it can be proved that $\mathcal{V}(k, k)=0$.

If $\mathcal{V}(j, k) \neq 0$, then the following holds:

$$
F(|\mathcal{V}(j, k)|)=F(|\mathcal{V}(T j, T k)|) \leq F\left(\frac{1}{2}(|\mathcal{V}(j, T j)|+|\mathcal{V}(k, T k)|)\right)-\tau
$$

and it is not well defined. Therefore, $\mathcal{V}(j, k)=0$.

It follows that $j=k$, and so the fixed point is unique.

To support our results, we give the next example. 
Example 6 Let $\mho=(-\infty, 0]$ and define the function $\mathcal{V}: \mho \times \mho \rightarrow \mathbb{R}$ by

$$
\mathcal{V}(j, k)= \begin{cases}|j-k|+1, & \text { if } j \neq k, \\ \max \{j, k\}, & \text { if } j=k .\end{cases}
$$

According to [21], the pair $(\mho, \mathcal{V})$ is a complete dualistic partial metric space. Consider the mapping $T: \mho \rightarrow \mho$ given as

$$
T j= \begin{cases}-1, & j \in(-\infty,-4], \\ -\frac{1}{2}, & j \in(-4,0] .\end{cases}
$$

We claim that $T$ fulfills the conditions of Theorem 2 for $\tau=\ln \frac{3}{2}$ and $F(t)=\ln t$.

We have to analyze several cases.

Case I. $j \neq k$ with $j, k \in(-4,0]$.

We note that $\mathcal{V}(T j, T k)=\mathcal{V}\left(-\frac{1}{2},-\frac{1}{2}\right)=-\frac{1}{2}$. Also,

$$
\begin{aligned}
\ln \frac{3}{2}+\ln |\mathcal{V}(T j, T k)| & =\ln \frac{3}{2}+\ln \left|-\frac{1}{2}\right| \\
& =\ln \frac{3}{4} \\
& \leq \ln \frac{\left|\mathcal{V}\left(j,-\frac{1}{2}\right)\right|+\left|\mathcal{V}\left(k,-\frac{1}{2}\right)\right|}{2} \\
& =\ln \frac{|\mathcal{V}(j, T j)|+|\mathcal{V}(k, T k)|}{2},
\end{aligned}
$$

and so it behaves accordingly.

Case II. $j \neq k$ with $j, k \in(-\infty,-4]$.

Here, we have

$$
\begin{aligned}
\ln \frac{3}{2}+\ln |\mathcal{V}(T j, T k)| & =\ln \frac{3}{2}+\ln |\mathcal{V}(-1,-1)| \\
& =\ln \frac{3}{2} \\
& \leq \ln \frac{|\mathcal{V}(j,-1)|+|\mathcal{V}(k,-1)|}{2} \\
& =\ln \frac{|\mathcal{V}(j, T j)|+|\mathcal{V}(k, T k)|}{2},
\end{aligned}
$$

and the relation is also checked.

Case III. $j \neq k$ with $j \in(-\infty,-4], k \in(-4,0]$ and vice versa.

In this case, we have $\mathcal{V}(T j, T k)=\mathcal{V}\left(-1,-\frac{1}{2}\right)=\frac{3}{2}$. Also,

$$
\begin{aligned}
\ln \frac{3}{2}+\ln |\mathcal{V}(T j, T k)| & =\ln \frac{3}{2}+\ln \left|\frac{3}{2}\right| \\
& =\ln \frac{9}{4}
\end{aligned}
$$




$$
\begin{aligned}
& \leq \ln \frac{|\mathcal{V}(j,-1)|+\left|\mathcal{V}\left(k, \frac{-1}{2}\right)\right|}{2} \\
& =\ln \frac{|\mathcal{V}(j, T j)|+|\mathcal{V}(k, T k)|}{2}
\end{aligned}
$$

Case IV. $j=k \in(-4,0]$.

We have

$$
\begin{aligned}
\ln \frac{3}{2}+\ln |\mathcal{V}(T j, T k)| & =\ln \frac{3}{2}+\ln \left|-\frac{1}{2}\right| \\
& =\ln \frac{3}{4} \\
& \leq \ln \frac{\left|\mathcal{V}\left(j,-\frac{1}{2}\right)\right|+\left|\mathcal{V}\left(k,-\frac{1}{2}\right)\right|}{2} \\
& =\ln \frac{|\mathcal{V}(j, T j)|+|\mathcal{V}(k, T k)|}{2},
\end{aligned}
$$

so, this case is also true.

Case V. $j=k \in(-\infty,-4]$.

In this case, one writes

$$
\begin{aligned}
\ln \frac{3}{2}+\ln |\mathcal{V}(T j, T k)| & =\ln \frac{3}{2}+\ln |\mathcal{V}(-1,-1)| \\
& =\ln \frac{3}{2} \\
& \leq \ln \frac{|\mathcal{V}(j,-1)|+|\mathcal{V}(k,-1)|}{2} \\
& =\ln \frac{|\mathcal{V}(j, T j)|+|\mathcal{V}(k, T k)|}{2} .
\end{aligned}
$$

The inequality in Theorem 2 is fulfilled. By applying this result, we get that $T$ has a unique fixed point, which is $j=-\frac{1}{2}$.

On the other hand, it can be easily verified that the inequality in Theorem 1 is not satisfied in the case $j=k=0$.

\section{Application to the boundary value problem}

In this section, we apply Theorem 1 to obtain the existence of the solution to the following boundary value problem. For more details on the applications of $\mathrm{BCP}$, readers are referred to $[41,42]$.

$$
\left\{\begin{array}{l}
-\frac{d^{2} \ell}{d l^{2}}=g^{\prime}(\dot{l}, \ell(l)), \quad l \in[0,1], \\
\ell(0)=\ell(1)=0,
\end{array}\right.
$$

where $g^{\prime}:[0,1] \times \mathbb{R} \longrightarrow \mathbb{R}$ is a continuous mapping. The Green function associated with the boundary value problem (4.1) is defined by

$$
V(\dot{l}, \breve{h})= \begin{cases}\check{l}(1-\check{h}), & 0 \leq \grave{l} \leq \check{h} \leq 1, \\ \breve{h}(1-\check{l}), & 0 \leq \check{h} \leq \grave{l} \leq 1 .\end{cases}
$$


Let $C[0,1]$ be the space of all continuous mappings defined on $[0,1]$. Let $\mathcal{C}=(C[0,1], \mathbb{R})$. Define the mapping $\mathcal{V}: \mathcal{C} \times \mathcal{C} \rightarrow \mathbb{R}$ by

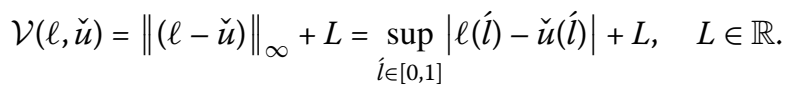

The pair $(\mathcal{C}, \mathcal{V})$ is a complete dualistic partial metric space. The associated operator $T$ : $\mathcal{C} \rightarrow \mathcal{C}$ to the boundary value problem (4.1) has the following form:

$$
T \ell(\dot{l})=\int_{0}^{1} V(\dot{l}, \check{h}) \dot{g}(\check{h}, \ell(\check{h})) d \check{h}
$$

for all $l \in[0,1]$. Note that problem (4.1) has a solution iff the operator $T$ has a fixed point.

Theorem 3 Let $\mathcal{C}=C([0,1], \mathbb{R})$ and suppose that the mapping $g:[0,1] \times \mathcal{C} \rightarrow \mathbb{R}$ satisfies

$$
|\grave{g}(\dot{l}, \ell)-\dot{g}(\grave{l}, \breve{u})| \leq 8 \ln \left(\frac{e^{e^{-\tau} \omega}}{\rho}\right)
$$

for all $l \in[0,1], \ell, \breve{u} \in \mathcal{C}, \omega=|\mathcal{V}(\ell, \breve{u})|, \rho>0$, and $\tau>0$. Then boundary value problem (4.1) has a solution.

Proof We note that $q(\bar{l}) \in\left(C^{2}[0,1], \mathbb{R}\right)$ (say) is a solution of (4.1) if and only if $q(\dot{l}) \in \mathcal{C}$ is a solution of integral equation (4.2). The solution of (4.2) is given by the fixed point of $T$, i.e., $q(\bar{l})=T(q(\bar{l}))$.

Let $\ell, \breve{u} \in \mathcal{C}$ and $l \in[0,1]$, by (4.3), we get

$$
\begin{aligned}
& |T \ell(\grave{l})-T \check{u}(\bar{l})|=\left[\left|\int_{0}^{1} V(\bar{l}, \check{h})[\dot{g}(\check{h}, \ell(\check{h}))-\dot{g}(\check{h}, \check{u}(\check{h}))] d \check{h}\right|\right] \\
& \leq\left[\int_{0}^{1} V(\grave{l}, \check{h})|\dot{g}(\check{h}, \ell(\check{h}))-\dot{g}(\check{h}, \check{u}(\check{h}))| d \check{h}\right] \\
& \leq\left[8 \int_{0}^{1} V(\dot{l}, \check{h}) \ln \left(\frac{e^{e^{-\tau} \omega}}{\rho}\right) d \check{h}\right] \\
& \leq\left[8 \int_{0}^{1} V(\dot{l}, \check{h}) \ln \left(\frac{e^{e^{-\tau} \omega}}{\rho}\right) d \check{h}\right] \\
& =8 \ln \left(\frac{e^{e^{-\tau} \omega}}{\rho}\right)\left(\sup _{\grave{l} \in[0,1]}\left[\int_{0}^{1} V(\grave{l}, \check{h}) d \check{h}\right]\right) \text {. }
\end{aligned}
$$

Since $\int_{0}^{1} V(\dot{l}, \check{h}) d \check{h}=-\frac{\hat{l}^{2}}{2}+\frac{\hat{l}}{2}$ for all $l \in[0,1]$, we have $\sup \left[\int_{0}^{1} V(\grave{l}, \check{h}) d \check{h}\right]=\frac{1}{8}$, which implies that

$$
\begin{aligned}
|\mathcal{V}(T(\ell), T(\check{u}))| & =\sup _{i \in[0,1]}|T \ell(\bar{l})-T \check{u}(\bar{l})|+L \leq e^{-\tau} \omega ;(L=\ln (\rho)) \\
& =e^{-\tau}(|\mathcal{V}(\ell, \check{u})|) .
\end{aligned}
$$


Taking $\ln$ on both sides, we have

$$
\ln |\mathcal{V}(T(\ell), T(\check{u}))| \leq \ln e^{-\tau}(|\mathcal{V}(\ell, \check{u})|) .
$$

Define $F(\ell)=\ln (\ell)$ for all $\ell \in \mathcal{C}$, and simplifying the last inequality, we have

$$
\tau+F(|\mathcal{V}(T(\ell), T(\check{u}))|) \leq F(|\mathcal{V}(\ell, \breve{u})|)
$$

Hence, the application of Theorem 1 ensures that $T$ has at least one fixed point $q(\dot{l}) \in \mathcal{C}$, that is, $T(q(\dot{l})=q(\dot{l})$ which is a solution of (4.2).

\section{Conclusion}

This paper has extended the classical $F$-contraction from the case $d(j, k)>0$ to $\mathcal{V}(j, k) \neq 0$. There are self-mappings defined on dualistic partial metric spaces which have no fixed points, but satisfy contractive conditions given in [24, 33, 35,37]. However, such mappings do not satisfy (3.1) and (3.7). Thus, contractive conditions (3.1) and (3.7) are refinements of the ordinary (Banach type and Kannan type) $F$-contractions. This paper has also presented a new method to show the existence of fixed points of $F$-contractions in dualistic partial metric spaces.

Acknowledgements

Not applicable.

Funding

Not applicable.

Availability of data and materials

Not applicable.

\section{Declarations}

\section{Competing interests}

The authors declare that they have no competing interests.

\section{Authors' contributions}

The authors equally conceived of the study, participated in its design and coordination, drafted the manuscript, participated in the sequence alignment, and read and approved the final manuscript.

\section{Author details}

${ }^{1}$ Department of Mathematics, Allama Iqbal Open University, H-8, Islamabad 44000, Pakistan. ${ }^{2}$ Institut Supérieur d'Informatique et des Techniques de Communication, Université de Sousse, H. Sousse 4000, Tunisia. ${ }^{3}$ China Medical University Hospital, China Medical University, Taichung, 40402, Taiwan. ${ }^{4}$ Department of Mathematics and Applied Mathematics, Sefako Makgatho Health Sciences University, Ga-Rankuwa, South Africa. ${ }^{5}$ Research Institute for Natural Sciences, Hanyang University, Seoul 04763, Korea. ${ }^{6}$ Department of Mathematics, International Islamic University, Islamabad 44000, Pakistan. ${ }^{7}$ Department of Mathematics, Uşak University, Uşak 64000, Turkey. ${ }^{8}$ Department of Mathematics, University of Seoul, Seoul 02504, Korea.

\section{Publisher's Note}

Springer Nature remains neutral with regard to jurisdictional claims in published maps and institutional affiliations.

Received: 9 February 2021 Accepted: 19 October 2021 Published online: 06 November 2021

\section{References}

1. Sedghi, S., Shobe, N., Zhou, H.: A common fixed point theorem in D*-metric spaces. Fixed Point Theory Appl. 2007, Article ID 27906 (2007)

2. Klim, D., Wardowski, D.: Fixed points of dynamic processes of set-valued F-contractions and application to functional equations. Fixed Point Theory Appl. 2015, 22 (2015)

3. Jleli, M., Samet, B.: On a new generalization of metric spaces. J. Fixed Point Theory Appl. 20, 128 (2018) 
4. Karapinar, E., Czerwik, S., Aydi, H.: $(\alpha, \psi)$-Meir-Keeler contraction mappings in generalized $b$-metric spaces. J. Funct. Spaces 2018, Article ID 3264620 (2018)

5. Mustafa, Z., Sims, B.: A new approach to generalized metric spaces. J. Nonlinear Convex Anal. 7, 289-297 (2006)

6. Parvaneh, V., Haddadi, M.R., Aydi, H.: On best proximity point results for some type of mappings. J. Funct. Spaces 2020, Article ID 6298138 (2020)

7. Suzuki, T.: Fixed point theorems for single- and set-valued $F$-contractions in $b$-metric spaces. J. Fixed Point Theory Appl. 20, 35 (2018)

8. Ali, M.U., Aydi, H., Alansari, M.: New generalizations of set valued interpolative Hardy-Rogers type contractions in b-metric spaces. J. Funct. Spaces 2021, Article ID 6641342 (2021)

9. Alqahtani, B., Fulga, A., Karapinar, E., Rakočević, V.: Correction to: contractions with rational inequalities in the extended b-metric space. J. Inequal. Appl. 2019, 229 (2019)

10. Afshari, H., Karapinar, E.: A discussion on the existence of positive solutions of the boundary value problems via $\psi$-Hilfer fractional derivative on b-metric spaces. Adv. Differ. Equ. 2020, 616 (2020)

11. Sedghi, S., Shobe, N., Aliouche, A.: A generalization of fixed point theorem in S-metric spaces. Mat. Vesn. 64, 258-266 (2012)

12. Matthews, S.G.: Partial metric topology. Ann. N.Y. Acad. Sci. 728, 183-197 (1995)

13. Bukatin, M., Kopperman, R., Matthews, S., Pajoohesh, H.: Partial metric spaces. Am. Math. Mon. 116(8), 708-718 (2009)

14. Han, S., Wu, J., Zhang, D.: Properties and principles on partial metric spaces. Topol. Appl. 230, 77-98 (2017)

15. Aydi, H., Karapinar, E., Shatanawi, W.: Coupled fixed point results for $(\psi, \varphi)$-weakly contractive condition in ordered partial metric spaces. Comput. Math. Appl. 62, 4449-4460 (2011)

16. Nazam, M., Aydi, H., Noorani, M.S., Qawaqneh, H.: Existence of fixed points of four maps for a new generalized F-contraction and an application. J. Funct. Spaces 2019, Article ID 5980312 (2019)

17. Karapinar, E., Agarwal, R.P., Aydi, H.: Interpolative Reich-Rus-Ćirić type contractions on partial metric spaces. Mathematica 6, 256 (2018)

18. O'Neill, S.J.: Partial metric, valuations and domain theory. Ann. N.Y. Acad. Sci. 806, 304-315 (1996)

19. Oltra, S., Valero, O.: Banach's fixed point theorem for partial metric spaces. Rend. Ist. Mat. Univ. Trieste 36, 17-26 (2004)

20. Nazam, M., Arshad, M., Abbas, M.: Some fixed point results for dualistic rational contractions. Appl. Gen. Topol. 17, 199-209 (2016)

21. Nazam, M., Aydi, H., Mukheimer, A., Arshad, M., Riaz, R.: Fixed point results for dualistic contractions with an application. Discrete Dyn. Nat. Soc. 2020, Article ID 6428671 (2020)

22. Nazam, M., Park, C., Arshad, M., Mahmood, H.: On a fixed point theorem with application to functional equations. Open Math. 17, 1724-1736 (2019)

23. Pitea, A.: Best proximity results on dualistic partial metric spaces. Symmetry 11, 306 (2019)

24. Wardowski, D.: Fixed points of a new type of contractive mappings in complete metric spaces. Fixed Point Theory Appl. 2012, 94 (2012)

25. Karapinar, E., Fulga, A., Agarwal, R.P.: A survey: F-contractions with related fixed point results. J. Fixed Point Theory Appl. 22,69 (2020)

26. Agarwal, R.P., Aksoy, U., Karapinar, E., Erhan, I.M.: F-Contraction mappings on metric-like spaces in connection with integral equations on time scales. Rev. R. Acad. Cienc. Exactas Fís. Nat., Ser. A Mat. 114, 147 (2020)

27. Aydi, H., Karapinar, E., Yazidi, H.: Modified F-contractions via alpha-admissible mappings and application to integral equations. Filomat 31, 1141-1148 (2017)

28. Acar, O., Altun, I.: Multivalued F-contractive mappings with a graph and some fixed point results. Publ. Math. (Debr.) $88,305-317(2016)$

29. Ali, M.U., Kamran, T:: Multivalued F-contractions and related fixed point theorems with an application. Filomat 30, 3779-3793 (2016)

30. Durmaz, G., Minak, G., Altun, I.: Fixed points of ordered F-contractions. Hacet. J. Math. Stat. 45, 15-21 (2016)

31. Nazam, M., Hussain, N., Hussain, A., Arshad, M.: Fixed point theorems for weakly admissible pair of $F$-contractions with application. Nonlinear Anal., Model. Control 24, 898-918 (2019)

32. Hammad, H.A., Aydi, H., Mlaiki, N.: Contributions of the fixed point technique to solve the 2 D Volterra integral equations, Riemann-Liouville fractional integrals and Atangana-Baleanu integral operators. Adv. Differ. Equ. 2021, 97 (2021)

33. Öztürk, A.: A fixed point theorem for mappings with an F-contractive iterate. Adv. Theory Nonlinear Anal. Appl. 3, 231-236 (2019)

34. Mlaiki, N., Souayah, N., Abdeljawad, T., Aydi, H.: A new extension to the controlled metric type spaces endowed with a graph. Adv. Differ. Equ. 2012, 94 (2021)

35. Gulyaz-Ozyurt, S.: A note on Kannan type mappings with a F-contractive iterate. Results Nonlinear Anal. 2, 143-146 (2019)

36. Hammad, H.A., Aydi, H., Gaba, Y.U.: Exciting fixed point results on a novel space with supportive applications. J. Funct. Spaces 2021, Article ID 6613774 (2021)

37. Bedre, S.: Remarks on F-weak contractions and discontinuity at the fixed point. Adv. Theory Nonlinear Anal. Appl. 4 , 260-265 (2020)

38. Proinov, P.D.: Fixed point theorems for generalized contractive mappings in metric spaces. J. Fixed Point Theory Appl. $22,21(2020)$

39. Skof, F: Theoremi di punto fisso per applicazioni negli spazi metrici. Atti Accad. Sci. Torino, Cl. Sci. Fis. Mat. Nat. 111, 323-329 (1977)

40. Batra, R., Gupta, R., Sahni, P.: A new extension of Kannan contractions and related fixed point results. J. Anal. 28, 1143-1154 (2020)

41. Hazarika, B., Karapinar, E., Arab, R., Rabbani, M.: Metric-like spaces to prove existence of solution for nonlinear quadratic integral equation and numerical method to solve it. J. Comput. Appl. Math. 328, 302-313 (2018)

42. Alsulami, H., Galyaz, S., Karapinar, E., Erhan, I.: An Ulam stability result on quasi-b-metric-like spaces. Open Math. 14, 1087-1103 (2016) 